\title{
Increased knowledge predicts greater adherence to the Mediterranean diet in Greek adolescents
}

\author{
Paraskevi K Tsartsali, Janice L Thompson* and Russell Jago \\ The University of Bristol, Department of Exercise, Nutrition \& Health Sciences, Tyndall Avenue, Bristol BS8 ITP, UK
}

Submitted 25 July 2007: Accepted 2 May 2008: First published online 1 August 2008

\begin{abstract}
Objectives: To examine knowledge of and adherence to the Mediterranean dietary pattern (MDP) among Greek adolescents, assess associations between MDP knowledge and adherence with BMI, and determine socio-cultural factors predicting MDP compliance.

Design: Cross-sectional.

Setting: Greek adolescents aged 15-17 years.

Subjects: Two hundred adolescents (103 females, ninety-seven males) from six schools on the Greek island of Chios. The sampling procedure was similar for all schools; schools were randomly selected from different geographic areas and all municipalities. BMI was calculated from measured height and weight; participants completed four questionnaires assessing parents' socio-economic status and education, adolescents' perceived and actual MDP knowledge, past-week dietary habits, and MDP adherence.

Results: Participants' BMI indicated 64.5\% were normal weight and 35.5\% were overweight/obese (mean BMI $\left.23 \cdot 7(\mathrm{sD} 3 \cdot 8) \mathrm{kg} / \mathrm{m}^{2}\right)$. Over half had very poor MDP knowledge $(58 \cdot 5 \%)$ and adherence $(59 \cdot 5 \%)$; both perceived $(F=3.35$, $P=0.037)$ and actual MDP knowledge $(F=3 \cdot 45, P=0 \cdot 034)$ were significantly different across MDP adherence. Perceived MDP knowledge was positively correlated with vegetable consumption $(r=0 \cdot 185, P=0 \cdot 009)$; actual knowledge was negatively correlated with meat consumption $(r=-0 \cdot 191, P=0 \cdot 007)$. BMI was negatively correlated with family income $(r=-0 \cdot 202, P=0 \cdot 004)$, indicating higher BMI in less affluent households. Actual MDP knowledge was the only significant predictor of MDP adherence (standardized $\beta=0 \cdot 162, P=0 \cdot 030$ ) in a model accounting for $7 \cdot 3 \%$ of overall variance.

Conclusions: Greek adolescents reported consuming a more Westernized diet detached from the traditional MDP. Actual MDP knowledge and family income were important factors affecting MDP adherence and BMI, respectively. Promoting the traditional MDP among Greek adolescents and their families appears warranted.
\end{abstract}

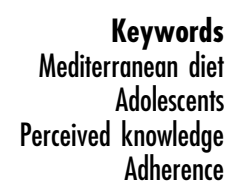

During the past three decades, many epidemiological studies conducted worldwide have presented evidence for the essential role of diet and specific dietary components in the prevention and control of morbidity and premature mortality resulting from chronic diseases ${ }^{(1)}$. The Mediterranean dietary pattern (MDP) has been widely promoted as a healthy eating model ${ }^{(2)}$. This type of eating pattern gained attention following the Seven Countries study conducted by Keys and Grande ${ }^{(3)}$, as they reported that Mediterranean countries following this eating pattern had lower rates of CHD and certain types of cancers, and a longer life expectancy.

The MDP is characterized by an abundance of plant foods such as fruits, vegetables, non-refined cereals and products, potatoes, beans, nuts and seeds ${ }^{(4)}$. Olive oil is the principal source of fat and moderate consumption of fish, poultry, dairy products (mainly cheese and yoghurt) and eggs is common, as is a relatively low consumption of red meat. Wine is consumed in low to moderate amounts, normally with meals ${ }^{(4)}$.

Despite the growing scientific evidence that has followed the Seven Countries study about the MDP and its health benefits in both primary and secondary prevention of many chronic diseases such as obesity, CHD and type 2 diabetes mellitus ${ }^{(5-13)}$, the national average of foods consumed in Greece has deviated from the MDP recommendations and Greece has adopted a more Westernized profile. With the exception of maintaining a high olive oil consumption and adequate fruit and vegetable intake, this deviation includes a greatly increased consumption of total energy, animal products, lipids other than olive oil and refined sugar ${ }^{(14,15)}$. 
These changes in dietary patterns are also apparent among children and adolescents in many Mediterranean countries, including Greece. The abandonment of the traditional MDP and the Westernization of younger generations' dietary habits in these areas is thought to deteriorate their CVD risk profile ${ }^{(16-18)}$, and predicts an unfavourable CVD morbidity and mortality for this population in the foreseeable future ${ }^{(19)}$. A school-based intervention study in Greek children and adolescents found an increased prevalence of CVD risk factors among approximately one-third of the participants ${ }^{(20)}$.

The prevalence of obesity, which is considered one of the most important risk factors not only for CVD but also for other diseases such as diabetes and some cancers, has dramatically increased worldwide in children and adolescents $^{(21,22)}$. According to Lobstein and Frelut ${ }^{(21)}$, the results from the limited number of studies conducted in Greece have indicated that the prevalence of children's and adolescents' obesity in Greece is one of the highest in Europe. As obese children and adolescents are at high risk of becoming obese adults ${ }^{(23)}$ and the development of CVD has its roots in childhood and adolescence ${ }^{(24)}$, the implementation of therapeutic and preventive measures such as education to prevent and reduce obesity should be of high importance ${ }^{(19)}$.

The unfavourable health profile of children and adolescents in Greece and the fact that dietary habits are established during childhood and adolescence make nutrition education a necessity in school programmes ${ }^{(25)}$. Consequently, the aims of the present study were to examine MDP knowledge and adherence among Greek adolescents, to assess the associations between MDP knowledge and adherence with BMI, and to determine whether various socio-cultural factors predict MDP compliance.

\section{Subjects and methods}

\section{Recruitment and etbical approval}

In the present cross-sectional study, 222 adolescent students from six schools located on the Greek island of Chios were initially asked to participate; 201 (90.54\%) agreed to participate. One student did not complete the survey during the data collection period; thus the final sample size was 200 (103 females and ninety-seven males). Ages ranged from 15 to 17 years and the sampling procedure was similar for all six schools that were included in the study for valid cross-regional comparison. The six schools were randomly selected from different geographic areas and from all municipalities of Chios. All participating students and their parents received an information sheet about the study and written consent was obtained from both students and their parents prior to participation in the study. Ethical approval was obtained from the School of Applied Community and Health Studies ethics committee at the University of Bristol.

\section{Measurements}

Anthropometric measurements were taken for each participant. Weight was measured to an accuracy of $0.01 \mathrm{~kg}$ with the use of a digital scale on participants without shoes and outer garments removed to leave only trousers or skirt and a T-shirt. Height was measured to the nearest $0.01 \mathrm{~cm}$ with the use of a measuring tape against a wall with the participants shoeless and in standing position with their shoulders relaxed and arms hanging freely. Participants' BMI was calculated by dividing weight $(\mathrm{kg})$ by the square of height $\left(\mathrm{m}^{2}\right)$.

\section{Questionnaires}

Four questionnaires were given to the participants for completion in the classroom: (i) a questionnaire on personal characteristics, socio-economic status (SES) and parents' education level; (ii) a twenty-item questionnaire based on the principles of the Mediterranean diet pyr$\operatorname{amid}^{(26)}$ for the assessment of adolescents' perceived and actual knowledge about the MDP and its health outcomes; (iii) an FFQ for the assessment of their past-week dietary habits ${ }^{(27)}$; and (iv) a brief (sixteen-item) questionnaire (KIDMED) for assessment of their adherence to the MDP that has been successfully used among children and adolescents in Spain ${ }^{(28-30)}$.

\section{Statistical analysis}

Means and percentages were calculated for demographic characteristics. Means and standard deviations for servings per week were calculated for all food group data. For this purpose adolescents' answers relative to their past-week food intake were grouped according to the Mediterranean diet pyramid as follows: meat, other meattype foods (e.g. allantika, meat soup, mousaka), poultry, sweets, potatoes, fish, dairy products, fruits, vegetables, non-refined cereals and bread products, nuts, olives, eggs and pulses. BMI groups were calculated according to the cut-off points for adolescents' BMI defined by the International Obesity Taskforce (IOTF) ${ }^{(31)}$.

Three categories were used to define adolescents' MDP adherence according to the KIDMED questionnaire ${ }^{(28-30)}$ : (i) $\leq 3$ points was defined as very poor adherence; (ii) 4-7 points was defined as medium adherence; and (iii) $\geq 8$ points was defined as high adherence.

Four categories were used to define adolescents' knowledge about MDP recommendations: (i) $\leq 5$ correct answers was defined as very poor knowledge; (ii) 6-10 correct answers was defined as poor knowledge; (iii) 11-15 correct answers was defined as medium knowledge; and (iv) 16-20 right answers was defined as high knowledge of the MDP and its health benefits. The first question of the MDP knowledge questionnaire assessed each adolescent's perceived knowledge (\%) about the MDP.

Pearson correlations were calculated between participants' descriptive characteristics, perceived MDP knowledge, actual MDP knowledge and MDP compliance. 
Pearson correlations were also calculated between perceived MDP knowledge, actual MDP knowledge, MDP compliance and the consumption of each food group. One-way ANOVA with associated post hoc Tukey tests were used to examine whether compliance with the MDP differed by participants' BMI, perceived MDP knowledge or actual MDP knowledge. All of these analyses were performed using the Statistical Package for the Social Sciences statistical software package version $14 \cdot 0$ (SPSS Inc., Chicago, IL, USA).

A linear regression model that controlled for the clustering of participants within schools was then performed using the XTREG procedure in the STATA statistical software package version 9.0 (Stata Corporation, College Station, TX, USA) to determine whether actual or perceived MDP knowledge predicted MDP compliance. The model included MDP compliance as continuous variable, with actual knowledge and perceived knowledge as independent variables while controlling for gender, age, BMI and family income. The associated within-group and between-group $R^{2}$ values for each model were obtained as was the overall $R^{2}$, which is comparable to the $R^{2}$ obtained from non-clustered models. Finally, a logistic regression model was run to determine whether actual MDP knowledge, perceived knowledge, gender, age, BMI or family income predicted whether participants had reasonable ( 4 or higher) or poor (3 or lower) MDP compliance. The model was run using the XTLOGIT command in STATA and controlled for the clustering of participants in schools. Alpha was set at $P<0.05$ for all analyses.

\section{Results}

Descriptive statistics for participant characteristics are shown in Table 1 . The sample was $52 \%$ female and predominately from lower- to middle-income households. Participants were $15 \cdot 8$ (SD 0.6 ) years of age with a mean BMI of $23 \cdot 7$ (SD $3 \cdot 8) \mathrm{kg} / \mathrm{m}^{2}$. Classification by the IOTF criteria indicated that $129(64.5 \%)$ were normal weight with fifty-one $(25 \cdot 5 \%)$ overweight and twenty obese $(10 \cdot 0 \%)$.

Descriptive statistics for the servings per week of key foods and adherence to the MDP are shown in Table 2. Participants were consuming $17 \cdot 1$ servings of fruits and 16.7 servings of vegetables weekly. This is approximately equivalent to $4 \cdot 8$ servings of fruits and vegetables daily. Analysis of the MDP questionnaire indicated that 119 (59.5\%) of the participants had very poor MDP adherence, with 117 (58.5\%) possessing poor or very poor actual knowledge. ANOVA indicated no difference in the BMI of participants with low, medium or high MDP adherence $(F=0 \cdot 60, \operatorname{df}(2,1), P=0 \cdot 545)$. There was a significant difference in perceived knowledge by MDP adherence $(F=3 \cdot 35, \mathrm{df}(2,1), P=0 \cdot 037)$ but there were no significant comparisons between the three adherence
Table 1 Descriptive statistics (frequencies and percentages, means and standard deviations) for participant characteristics: adolescents aged $15-17$ years, Chios, Greece

\begin{tabular}{|c|c|c|}
\hline & $n$ & $\%$ \\
\hline \multicolumn{3}{|l|}{ Gender } \\
\hline Boys & 97 & 48.5 \\
\hline Girls & 103 & $51 \cdot 5$ \\
\hline \multicolumn{3}{|l|}{ Father's education } \\
\hline Less than 6 years & 26 & $13 \cdot 0$ \\
\hline $6-12$ years & 58 & $29 \cdot 0$ \\
\hline More than 12 years & 116 & $58 \cdot 0$ \\
\hline \multicolumn{3}{|l|}{ Mother's education } \\
\hline Less than 6 years & 37 & $18 \cdot 5$ \\
\hline $6-12$ years & 89 & $44 \cdot 5$ \\
\hline More than 12 years & 74 & $37 \cdot 0$ \\
\hline \multicolumn{3}{|l|}{ Father's working status } \\
\hline Not working/retired & 16 & $8 \cdot 0$ \\
\hline Working & 184 & $92 \cdot 0$ \\
\hline \multicolumn{3}{|l|}{ Mother's working status } \\
\hline House holding/retired & 98 & $49 \cdot 0$ \\
\hline Working & 102 & $51 \cdot 0$ \\
\hline \multicolumn{3}{|l|}{ Family income } \\
\hline Low & 92 & $46 \cdot 0$ \\
\hline Medium & 96 & $48 \cdot 0$ \\
\hline \multirow[t]{2}{*}{ High } & 12 & $6 \cdot 0$ \\
\hline & Mean & SD \\
\hline Age (years) & $15 \cdot 8$ & 0.6 \\
\hline BMl $\left(\mathrm{kg} / \mathrm{m}^{2}\right)$ & $23 \cdot 7$ & $3 \cdot 8$ \\
\hline
\end{tabular}

Table 2 Descriptive statistics (means and standard deviations, frequencies and percentages) for consumption of individual food groups, adherence to and knowledge of the Mediterranean diet pattern (MDP): adolescents aged 15-17 years, Chios, Greece

\begin{tabular}{|c|c|c|}
\hline & Mean & SD \\
\hline \multicolumn{3}{|l|}{ Food groups (servings/week) } \\
\hline Meat & $7 \cdot 79$ & $4 \cdot 62$ \\
\hline Meat food & $5 \cdot 37$ & 4.09 \\
\hline Poultry & $2 \cdot 34$ & $2 \cdot 17$ \\
\hline Fish & $3 \cdot 77$ & 3.05 \\
\hline Dairy products & $18 \cdot 92$ & $10 \cdot 24$ \\
\hline Non-refined cereals and products & $28 \cdot 80$ & $11 \cdot 67$ \\
\hline Pulses & 2.94 & $2 \cdot 39$ \\
\hline Fruits & $17 \cdot 10$ & $12 \cdot 78$ \\
\hline Vegetables & $16 \cdot 72$ & $10 \cdot 95$ \\
\hline Potatoes & $5 \cdot 00$ & $3 \cdot 19$ \\
\hline Sweets & $16 \cdot 10$ & $11 \cdot 87$ \\
\hline Eggs & $2 \cdot 31$ & $1 \cdot 84$ \\
\hline Olives & $1 \cdot 48$ & $1 \cdot 84$ \\
\hline \multirow[t]{2}{*}{ Nuts } & $1 \cdot 70$ & $1 \cdot 67$ \\
\hline & $n$ & $\%$ \\
\hline \multicolumn{3}{|l|}{ Adherence to MDP } \\
\hline Very poor & 119 & $59 \cdot 5$ \\
\hline Medium & 56 & $28 \cdot 0$ \\
\hline High & 25 & $12 \cdot 5$ \\
\hline \multicolumn{3}{|l|}{ Perceived knowledge of MDP } \\
\hline Know nothing & 19 & $9 \cdot 5$ \\
\hline Know a few recommendations & 84 & $42 \cdot 0$ \\
\hline Know many recommendations & 88 & $44 \cdot 0$ \\
\hline Know a lot of recommendations & 9 & 4.5 \\
\hline \multicolumn{3}{|l|}{ Actual knowledge about MDP } \\
\hline Very poor & 6 & $3 \cdot 0$ \\
\hline Poor & 111 & $55 \cdot 5$ \\
\hline Medium & 82 & $41 \cdot 0$ \\
\hline High & 1 & 0.5 \\
\hline
\end{tabular}


Table 3 Regression model predicting compliance to the Mediterranean diet pattern: adolescents aged 15-17 years, Chios, Greece

\begin{tabular}{lrrrrr}
\hline Variable & Coefficient & SE & Standardized $\beta$ & $Z$ & \multicolumn{1}{c}{$Z$} \\
\hline Actual knowledge & 0.329 & 0.15 & 0.162 & 2.17 & 0.030 \\
Perceived knowledge & 0.844 & 0.48 & 0.129 & 1.75 & 0.079 \\
Gender & -1.194 & 0.67 & -0.125 & -1.78 & 0.075 \\
Age & 0.388 & 0.56 & 0.048 & 0.69 & 0.491 \\
BMl & -0.061 & 0.09 & -0.048 & -0.67 & 0.506 \\
Family income & -0.268 & 0.58 & -0.034 & -0.47 & 0.642 \\
\multicolumn{1}{c}{ Within school $R^{2}=0.0725$} & Between school $R^{2}=0.031$ & 0 Overall $R^{2}=0.073$ \\
\hline
\end{tabular}

groups. There was a significant difference in actual knowledge of the three adherence groups $(F=3 \cdot 45$, df(2,1), $P=0 \cdot 034)$, with Tukey post hoc tests indicating a difference between the low and medium adherence groups that approached significance $(P=0 \cdot 065)$.

Correlations were calculated between participant characteristics, perceived MDP knowledge, actual knowledge and adherence with food group consumption. A number of significant correlations (all $P<0.05$ ) were found. Gender was negatively associated with consumption of meat $(r=-0 \cdot 245)$, fish $(r=-0 \cdot 141)$, non-refined cereals $(r=-0 \cdot 351)$, sweets $(r=-0 \cdot 150)$ and nuts $(r=-0 \cdot 252)$, indicating lower consumption of these foods by boys. Mother's work status was negatively correlated with meat $(r=-0 \cdot 158)$, non-refined cereals $(r=-0 \cdot 237)$, pulses $(r=-0 \cdot 150)$, fruits $(r=-0 \cdot 190)$, vegetables $(r=-0 \cdot 153)$, sweets $(r=-0 \cdot 217)$ and nuts $(r=-0 \cdot 162)$, indicating that participants whose mother did not work consumed more of these foods. Perceived MDP knowledge was positively correlated with vegetable consumption $(r=0 \cdot 185, P=0 \cdot 009)$ while actual knowledge was negatively correlated with meat consumption $(r=-0 \cdot 191, P=0 \cdot 007)$. BMI was negatively correlated with family income $(r=-0 \cdot 202, P=0 \cdot 004)$, indicating higher BMI in less affluent households.

The results of the regression model predicting MDP adherence are shown in Table 3. Actual knowledge was the only significant predictor of MDP adherence (standardized $\beta=0 \cdot 162, P=0 \cdot 030)$ in a model that accounted for $7 \cdot 3 \%$ of the variance within schools, $3 \cdot 1 \%$ of the variance between schools and $7 \cdot 3 \%$ of the overall variance.

\section{Discussion}

The results indicate that $35 \cdot 5 \%$ of the 200 Chian adolescents participating in the present study were overweight or obese, and that more than half had very poor actual knowledge of, and adherence to, the MDP. There was no difference in BMI values between participants with low, medium or high MDP adherence, and actual knowledge was the only variable found to be a significant predictor of MDP adherence in this sample.

Many studies have presented strong evidence for the role of the Mediterranean diet in health promotion. The whole pattern apart from its individual nutrients is considered to be protective against several diseases ${ }^{(5,11,12,32)}$. Nevertheless, despite the existing evidence about its health benefits, the deterioration of the MDP is apparent in many studies conducted in Greece and other Mediterranean countries, not only among adult populations ${ }^{(11,17,33)}$ but also among younger generations ${ }^{(27,29,34,35)}$. The results of the past-week FFQ in the present study indicted a lower consumption of food groups such as non-refined products, vegetables, olives and nuts, and a higher intake of meats and sweets. This eating pattern indicates a lower intake of foods considered protective against CVD and some cancers ${ }^{(7,9)}$ and a higher intake of foods that could contribute to excessive energy intake and subsequent obesity. These results are consistent with data reported for fruit and vegetable intake in 11-year-old children in nine European countries (not including Greece); while fruit and vegetable intake varied widely across countries, total intake was less than currently recommended ${ }^{(36)}$. The results of the present study add to the growing body of literature indicating that Greek adolescents are no longer consuming a traditional MDP and the recommended servings of fruit and vegetables each day.

According to the theory of planned behaviour, knowledge is considered one of the most important predictors for food choices among adolescents ${ }^{(37)}$. In the present study perceived and actual knowledge were examined as was their potential association with MDP adherence. Although the direction of causality is not clear because of the cross-sectional design of the present study, regression findings have indicated that it is actual knowledge about the MDP that is key and appears to predict MDP adherence $(r=0 \cdot 2, \quad P=0 \cdot 005)$. Consequently, adolescents should be more aware about the MDP and its health benefits, as this may assist them in adopting a less Westernized way of eating that is lower in energy and saturated fat.

The findings of the present study suggest that prevention efforts need to focus on increasing actual MDP knowledge among Greek adolescents. According to Reynolds et al. ${ }^{(38)}$, knowledge of the Five-A-Day guideline for fruit and vegetable consumption was the key to increasing fruit and vegetable intake among elementaryschool children in the USA. These investigators also suggest that combining knowledge with goal setting is 
likely to be more effective. Schools are considered suitable places for the implementation of interventions ${ }^{(39)}$, and the results from studies conducted in Greece on school-based health promotion programmes show they are positively correlated with an increase in health knowledge ${ }^{(20,40,41)}$. Thus the implementation of a school-based nutrition education programme focusing on the MDP and its health benefits among Greek adolescents could be an essential step towards primary prevention of CVD and would be of substantial importance for future public health.

In the present study adolescents' BMI was negatively correlated with family income, indicating a higher BMI in adolescents living in less affluent households. These results are consistent with those found for adolescents living in countries such as the USA, UK and Australia, where children living in lower SES environments have higher BMI values ${ }^{(42-44)}$. There do not appear to be any published data on the relationship between SES and BMI among Greek adolescents. A study of Greek adults aged 18 to 87 years found that people living in lower and middle SES households had higher BMI ${ }^{(45)}$. This association between SES and BMI among Greek adults was mainly explained by lower physical activity levels and higher energy intakes in those of lower SES. As physical activity status and energy intake were not measured in the present study, it is not possible to determine the role they might play in the interaction between family income and BMI among Chian adolescents.

There are a number of limitations in the present study. The majority of data are self-reported and it is recognized that self-report measures of nutrient intake are subject to recall bias ${ }^{(46)}$. Additionally, the FFQ tool used in the present study for the assessment of adolescents' dietary habits provides information on habitual dietary patterns and can be used for assessment only of the frequency and not the actual quantity of the consumed foods. Another limitation is that the KIDMED index was developed for Spanish children and adolescents and has not been validated in Greek adolescents. However, there are no published tools to assess MDP adherence in Greek adolescents. For the purposes of the present study, it was felt that this tool was appropriate as it assesses adherence to general MDP principles that are widely applicable across various Mediterranean populations. Furthermore, the present study included a relatively small sample of adolescents from the Greek island of Chios. Although Chios is the fifth biggest island of Greece, its inhabitants (approximately 25000) account for only a small percentage of the entire Greek population. Consequently, these results may not be generalizable to all Greek adolescents. However, the use of previously validated questionnaires in this sample of Chian adolescents (a group that has yet to be studied) can be considered an important strength which gives validity to the outcomes of the present study.

In conclusion, our findings suggest that the dietary habits of Chian adolescents present a more Westernized profile and a detachment from the traditional MDP. Actual MDP knowledge and family income seem to be the most important factors affecting adherence to the MDP and adolescents' BMI, respectively. A National Nutrition Policy for Greece that promotes the traditional MDP among adolescents and their families combined with an active lifestyle would likely result in a more favourable health profile in the future.

\section{Acknowledgements}

We would like to thank the schools, participants and parents of Chios for their participation in and contributions to this study.

Sources of funding: This research was not funded by any source.

Conflict of interest declaration: None declared.

Authorship responsibilities: P.K.T. conceptualized the study, collected and analysed the data, and drafted the manuscript. J.L.T. participated in the conceptualization of the study, interpretation of results and drafting the manuscript. R.J. assisted in data analysis, interpretation of results and drafting the manuscript. All authors read and approved the final manuscript.

\section{References}

1. World Health Organization (2003) Diet, Nutrition and the Prevention of Chronic Diseases. Geneva: WHO.

2. Hu F (2003) The Mediterranean diet and mortality - olive oil and beyond. N Engl J Med 348, 2595-2596.

3. Keys A \& Grande F (1957) Dietary fat and serum cholesterol. Am J Public Health 47, 1520-1530.

4. Willett WC, Sacks F \& Trichopoulou A (1995) Mediterranean diet pyramid: a cultural model for health eating. Am J Clin Nutr 61, S1402-S1406.

5. Trichopoulou A, Costacou T, Bamia C \& Trichopoulos D (2003) Adherence to a Mediterranean diet and survival in a Greek population. N Engl J Med 348, 2599-2608.

6. Vecchia C (2004) Mediterranean diet and cancer. Public Health Nutr 7, 965-968.

7. Lorgeril M \& Salen P (2006) The Mediterranean-style diet for the prevention of cardiovascular diseases. Public Health Nutr 9, 118-123.

8. Perez-Jimenez F, Lopez-Miranda J, Pinillos MD et al. (2001) A Mediterranean and a high-carbohydrate diet improve glucose metabolism in healthy young persons. Diabetologia 44, 2038-2043.

9. Wahrburg U, Kratz M \& Cullen P (2002) Mediterranean diet, olive oil and health. Eur J Lipid Sci Technol 104, 698-705.

10. Mendez MA, Popkin BM, Jakszyn P et al. (2006) Adherence to a Mediterranean diet is associated with reduced 3-year incidence of obesity. J Nutr 136, 2934-2938.

11. Schröder H (2007) Protective mechanisms of the Mediterranean diet in obesity and type 2 diabetes. J Nutr Biochem 18, 149-160.

12. Giugliano D \& Esposito K (2005) Mediterranean diet and cardiovascular health. Ann N Y Acad Sci 1056, 253-260.

13. Trichopoulou A, Bamia C \& Trichopoulos D (2005) Mediterranean diet and survival among patients with 
coronary heart disease in Greece. Arch Intern Med $\mathbf{1 6 5}$ 929-935.

14. Alexandratos N (2006) The Mediterranean diet in a world context. Public Health Nutr 9, 111-117.

15. Balanza R, García-Lorda P, Pérez-Rodrigo C, Aranceta J, Bulló Bonet M \& Salas-Salvadó J (2007) Trends in food availability determined by the Food and Agriculture Organization's food balance sheets in Mediterranean Europe in comparison with other European countries. Public Health Nutr 10, 168-176.

16. Amorim Cruz JA (2000) Dietary habits and nutritional status in adolescents over Europe - Southern Europe. Eur J Clin Nutr 54, S29-S35.

17. Moschandreas J \& Kafatos A (1999) Food and nutrient intake of Greek (Cretan) adults. Recent data for food-based dietary guidelines in Greece. BrJ Nutr 81, S71-S76.

18. Roma-Giannikou E, Adamidis D, Gianniou M, Nikolara R \& Matsaniotis N (1997) Nutritional survey in Greek children: nutrient intake. Eur J Clin Nutr 51, 273-285.

19. Magkos F, Manios Y, Christakis G \& Kafatos AG (2005) Secular trends in cardiovascular risk factors among schoolaged boys from Crete, Greece, 1982-2002. Eur J Clin Nutr 59, 1-7.

20. Manios Y, Moschandreas J, Hatzis C \& Kafatos A (1999) Evaluation of a health and nutrition education program in primary school children of Crete over a three year period. Prev Med 28, 149-159.

21. Lobstein T \& Frelut ML (2003) Prevalence of overweight among children in Europe. Obes Rev 4, 195-200.

22. Guillame M \& Lissau I (2002) Epidemiology. In Child and Adolescent Obesity: Causes and Consequences, Prevention and Management, pp. 28-49 [W Burniat, T Cole, I Lissau and EME Poskitt, editors]. Cambridge: Cambridge University Press.

23. Dietz WH (1998) Health consequences of obesity in youth: childhood predictors of adult disease. Pediatrics 56, 796-809.

24. Moller JH, Taubert KA, Allen HD, Clark EB \& Lauer RM (1994) Cardiovascular health and disease in children: current status. Circulation 89, 923-930.

25. Hassapidou M, Fotiadou E, Maglara E \& Papadopoulou S (2006) Energy intake, diet composition, energy expenditure, and body fatness of adolescents in northern Greece. Obesity 14, 855-862.

26. Trichopoulou A (2004) Traditional Mediterranean diet and longevity in the elderly: a review. Public Health Nutr 7, 943-947.

27. Hassapidou NM \& Fotiadou E (2001) Dietary intakes and food habits of adolescents in northern Greece. Int J Food Sci Nutr 52, 109-116.

28. Serra-Majem L, Garcia-Closas R, Ribas L, Perez-Rodrigo C \& Aranceta J (2001) Food patterns of Spanish schoolchildren and adolescents: The enKid study. Public Health Nutr $\mathbf{4}$, $1433-1438$.

29. Serra-Majem L, Ribas L, Garcia A, Perez-Rodrigo C \& Aranceta J (2003) Nutrient adequacy and Mediterranean diet in Spanish school children and adolescents. Eur J Clin Nutr 57, S35-S39.

30. Serra-Majem L, Ribas L, Ngo J, Ortega RM, Garcia A, PerezRodrigo C \& Aranceta J (2004) Food, youth and the Mediterranean diet in Spain. Development of KIDMED,
Mediterranean diet quality index in children and adolescents. Public Health Nutr 7, 931-935.

31. Cole TJ, Bellizzi MC, Flegal KM \& Dietz W (2000) Establishing a standard definition for child overweight and obesity worldwide: international survey. BMJ 320, 1240-1246.

32. Trichopoulou A \& Vasilopoulou E (2000) Mediterranean diet and longevity. Br J Nutr 84, S205-S209.

33. Panagiotakos D, Chrysochoou C, Pitsavos C \& Stefanadis C (2006) Association between the prevalence of obesity and adherence to the Mediterranean diet: the ATTICA study. Nutrition 22, 449-456.

34. Yannakoulia M, Karayiannis D, Terzidou M, Kokkevi A \& Sidossis LS (2004) Nutrition-related habits of Greek adolescents. Eur J Clin Nutr 58, 580-586.

35. Leclercq C, Picinnelli R, Arcella D \& Le CD (2004) Food consumption and nutrient intake in a sample of Italian secondary school students: results from the INRAN-RM2001 food survey. Int J Food Sci Nutr 55, 265-277.

36. Yngve A, Wolf A, Poortvliet E et al. (2005) Fruit and vegetable intake in a sample of 11-year-old children in 9 European countries: the Pro Children Cross-Sectional Survey. Ann Nutr Metab 49, 236-245.

37. Backman DR, Haddad EH, Lee JW, Johnston PK \& Hodgkin GE (2002) Psychosocial predictors of healthful dietary behavior in adolescents. J Nutr Educ Behav 34, 184-193.

38. Reynolds KD, Bishop DB, Chou C-P, Xie B, Nebeling L \& Perry CL (2004) Contrasting mediating variables in two 5-a-day nutrition intervention programs. Prev Med 39, 882-893.

39. Kelleher C (1995) Health promotion: shades of Lewis Carroll. J Epidemiol Community Health 49, 1-4.

40. Manios Y, Moschandreas J, Hatzis C \& Kafatos A (2002) Health and nutrition education in primary schools of Crete: changes in chronic disease risk factors following a 6-year intervention programme. Br J Nutr 88, 315-324.

41. Kafatos I, Peponaras A, Linardakis M \& Kafatos A (2004) Nutrition education and Mediterranean diet: exploring the teaching process of a school based nutrition and media education project in Cretan primary schools. Public Health Nutr 7, 969-975.

42. Chen E \& Paterson LQ (2006) Neighborhood, family, and subjective socioeconomic status: how do they relate to adolescent health? Health Psychol 25, 704-714.

43. Wardle J, Henning Brodersen N, Cole TJ, Jarvis MJ \& Boniface DR (2006) Development of adiposity in adolescence: five year longitudinal study of an ethnically and socioeconomically diverse sample of young people in Britain. BMJ 332, 1130-1135.

44. O'Dea JA \& Wilson R (2006) Socio-cognitive and nutritional factors associated with body mass index in children and adolescents: possibilities for childhood obesity prevention. Health Educ Res 21, 796-805.

45. Manios Y, Panagiotakos DB, Pitsavos C, Polychronopoulos E \& Stefanadis C (2005) Implication of socio-economic status on the prevalence of overweight and obesity in Greek adults: the ATTICA study. Health Policy 74, 224-232.

46. Kristal AR, Andrilla HA, Koepsell TD, Diehr PH \& Cheadle A (1998) Dietary assessment instruments are susceptible to intervention-associated response set bias. J Am Diet Assoc 98, 40-43. 